\title{
WHIRLING EFFECT ON BEARINGS OF MARINE LINE- SHAFTS UNDER EXTERNAL EXCITATIONS
}

\author{
H.M.METWALLY ${ }^{*}, 0 . Y$. SHOULA $^{*}$
}

\author{
ABSTRACT
}

Due to the ecentricity of thrust force acting on propeller blades, : marine shafts experience whirl while rotating. This directly affects the reactions of the different supports, and consequently causes wear of shaft bearings. This paper is concerned with simplifying the concept for the shafting system in order to estimate the frequencies

of whirl and propeller tip deflections. The work is dealing with

both; three and four span systems with overhaged propeller. The problem is also studied for both mass and massless shafts. For massless shafts systems of any number of spans, general formulae for calculating the frequency of whirl and the deflection of propeller

- tip are proposed. For shaft systems of distributed mass, a procedure. is suggested to calculate frequencies as well as deflections at different supports.

\section{INTRODUCTION}

A shafting system is a line-shaft joining the propelsive plant at one end, with the propeller at the other end. This shaft is support-: ed by a number of bearings of different flexibilities. Such a system is subjected to the different forces coming from various sources of excitation directly or through the structure. The external moment variations applied on the actuating propeller and the shafting system will induce a whirling phenomenon. These would also cause

: stress variations on the system, whichwere illustrated mathematical-

: ly by Jasper and Rupp [1]. If the wirling frequencies are somewhat close in value to the frequencies of any of the excitation sources, one should expect resonance. Hence extra vibrational stresses not

: only on the propeller and tail shaft but also on the whole after part of the ship's hull. The latter type of vibrations always been the source of trouble to the senstive equipment installed on board ship and inconvenience to the crew.

Problems Arise As a Result of Whirling Phenomenon:

The dynamic nature of this phenomenon would cause additional fatigue:

: stresses in the propeller shaft and the tail shaft, especially at

the front end of the propeller key way. The bearing reactions would be also magnified causing the vibration of the after body of the : ship. The first bearing just forward of the stern tube would be

* Associate Professor, University of Alexandria, Faculty of Engineering, Mechanical Engineering Dept.

* Naval Architect \& Member of Royal Institution of Naval Architects. 
damaged in a wiped up and heated upper half, while the lower half is: seemed undamaged. In general the stern tube would suffer the most, leading to any or many of undesirable behaveiours, such as: premat-

urely worn lignum vitae staves in the after bearings, fracture of some of the bushes, a rapid wear and/or the deterioration of tail-: shaft, seizing of the metallic bushes, heavy cavitation and galvanic

: corrosion in the central annual space of the stern-tube, cavitation erosion marks could be traced on the tail-shaft in way of the after vitae bearing bush, last but not least, damage of the stern-tube as: a whole due to exceessive bearing reactions. These problems and many

: others could be experienced by marine engineers working on board ships or could be seen as illustrated in Solumsmoen [2] and Svenson[3]

$\vdots$

\section{PROPELLER EXCTTATIONS}

When a propeller is situated behind a ship's hull, it tends to be irregularly fed due to the non-uniform wake pattern, and the turbulent layer of the water streams affected by the after hull. In such case the torque caused by the propulsive plant when transmitted into. thrust at the propelier blades will induce variations of forces and :

: moments which consequently influence the behaviour of the shaft.

A'study by Frivold [4], on four and five bladed propellers showed : : that not only there are variations of torque and moments, with respect to the angles of rotation, but that there are also variations in the values of thrust creating horizontal and vertical forces together with eccentricties acting on the propeller shaft. These:

$\vdots$ variations in thrust with eccentricities are the cause of the whirling phenomenon or the so called "lateral vibrations".

: The frequencies of these forces are equal to the propeller blade rate -i.e. the propeller blade frequency-and its multiples-e.g. for a four bladed propeller running at $180 \mathrm{rpm}$, the frequencies for the first harmonics will be: $120 \mathrm{~Hz}$ for the fundamental harmonic and

: $240 \mathrm{~Hz}$ for the second harmonic。

\section{FREQUENCIES OF LATERAL VIBRATIONS}

The method used here is based on the known simple beam theory given by Timoshinko [5]. The calculations are considering the following : assumptions throughout the calculations:

The eccentric force acting on the blade is assumed of a max of $10 \%$ of the mean thrust, and is applied at a mean distance of eccentricity.

: The rotios between deflections and slopes at any point remain constant.

$\vdots$ The first support which is meant to be the long stern tube bearing is considered fixed relative to the overhanged propeller shaft.

: The shaft is considered elastic and massless relative to the mass of: propeller. Hence the static bending moment of each span is neglected.

: The effect of axial thrust force is neglected.

\section{A SYSTEM OF THREE MASSLESS SPANS}

: Consider the case of supported shaft as shown in Fig. (1-a) A thrust: moment $M$ is applied statically at the free end $A$, causing a deflection as shown in Fig. $(1-b)$.

To study the system, one can separate the three spans and examins each span individually and then relate the effect of each span, on Lits adjacent. 
(a)

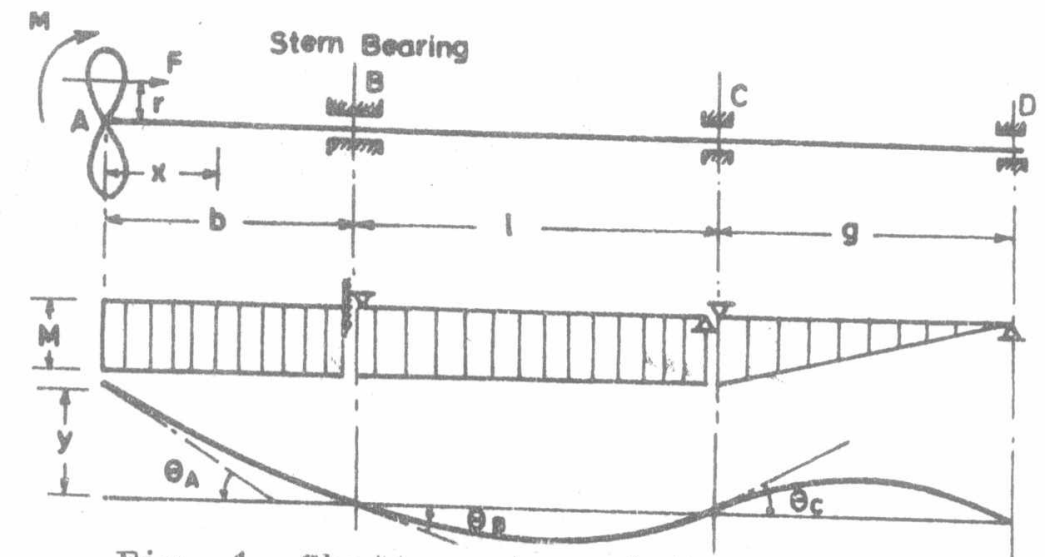

Fig. 1 Shatt system of three spans.

: Span CD

The bending moment, the slope and the deflection at any point $x$

(b)

(c)

$$
\begin{aligned}
& M_{x}=M \frac{g-x}{g} \\
& \theta_{x}=\frac{M}{E I}\left(x-\frac{x^{2}}{2 g}\right)+C_{1} \\
& y_{x}=\frac{M}{E I}\left(\frac{x^{2}}{2}-\frac{x^{3}}{6 g}\right)+C_{1} x+C_{2}
\end{aligned}
$$

where;

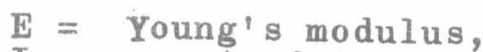

$\vdots \quad I=$ moment of inertia of shaft section.

$C_{1} \& C_{2}$ are constants of integration.

: Applying the boundary conditions for span CD, we get :

$$
\mathrm{C}_{2}=0 \text { and } \mathrm{C}_{1}=-\frac{\mathrm{M}}{\mathrm{EI}} \cdot \frac{\mathrm{g}}{3}
$$

: Thus, the slope equation of span CD becomes;

$$
\vdots \quad \theta=\frac{M}{E I}\left(x-\frac{x^{2}}{2 g}-\frac{g}{3}\right)
$$

The deflection equation for span CD becomes;

$$
\vdots
$$

$$
y=\frac{M}{E I}\left(\frac{x^{2}}{2}-\frac{x^{3}}{6 g}-\frac{g \cdot x}{3}\right)
$$

: At support $\mathrm{C}$ i.e. at $x=0$,

$$
\theta_{c}=\frac{-M}{E I} \frac{g}{3}, y_{c}=0
$$

Span BC

: Applying the general equations for bending moment, slope, and as (1), (2) \& (3) point $x$ from support $B$, we get the same relations

Applying the boundary conditions at point $C$, we get the general ¿..

slope and deflection equations, respectively; 


$$
\begin{aligned}
& & \theta_{\mathrm{BC}} & =\frac{\mathrm{M}}{\mathrm{EI}}\left(\mathrm{x}-\frac{1}{2}\right) \\
\vdots & & \mathrm{y}_{\mathrm{BC}} & =\frac{\mathrm{M}}{\mathrm{EI}}\left(\frac{\mathrm{x}^{2}}{2}-\frac{\mathrm{I} \cdot \mathrm{x}}{2}\right)
\end{aligned}
$$

At point B, we have

$$
: \quad \theta_{B}=\frac{M}{E I} \cdot \frac{1}{2} \quad(+ \text { Ve for convention })
$$

$$
\text { Positive in }+ \text { Ve clock wise rotation }
$$

: From the above discussion, one can say that the total slope at point. $B$ is the sum of the slopes to the two spans to the right BC \& CD. :

Thus from Eq. (6) \& (9), the total slope

$$
\vdots \quad \theta_{\mathrm{BB}}=\theta_{\mathrm{B}}+\theta_{\mathrm{C}}=\frac{\mathrm{M}}{\mathrm{EI}}\left(\frac{1}{2}-\frac{\mathrm{g}}{3}\right)
$$

\section{: Span AB}

If this span is assumed fixed at point B-Stern Tube Bearing the bending moment, slope, and deflection of any point $x$ measure from : : support A would be :

$$
\begin{array}{rlrl}
M_{x} & =M \\
\vdots & \theta_{x} & =\frac{M \cdot x}{E I}+C_{1}^{\prime \prime} \\
\vdots & y_{x} & =\frac{M x^{2}}{2 E I}+C_{1}^{\prime \prime}+C_{2}^{\prime \prime}
\end{array}
$$

Applying the boundary conditions, the general equations for slope : : and deflection at any point along span $\mathrm{AB}$, become;

$$
\begin{aligned}
\theta_{x} & =\frac{M}{E I}(x-b) \\
y_{x} & =\frac{M}{E I}\left(\frac{x^{2}}{2}-b \cdot x+\frac{b^{2}}{2}\right)
\end{aligned}
$$

At point $\mathbf{A}$

$$
\vdots \quad \theta_{\mathrm{A}}=\frac{\mathrm{bM}}{\mathrm{EI}}(+\mathrm{Ve} \text { for convention })
$$

The Equation of Motion

To lay down the equation of motion we assume that at all times the : : slope and moments are equal in value at both sides of each support.

Now for the separated span $A B$, the moments acting to the left of: : point B are :

Excitation periodic moment $=\mathrm{M}_{\mathrm{e}} \sin \mathrm{wt}$

: where : 
Inertia moment of the propller mass caused by the angular acceleration due to the slope $\theta_{B B}$ alone,

$$
=\ddot{\theta}_{\mathrm{BB}} \cdot\left(\mathrm{I}_{\mathrm{d}}+\mathrm{m}_{\mathrm{p}} \mathrm{b}^{2}\right)
$$

! where:

$$
\begin{aligned}
& I_{d}= \text { mass moment of inertia of propeller about a diameter } \\
& \text { corrected for the effect of entrained water, calculated } \\
& \text { as given by obrien [6]. } \\
& m_{p}= \text { the propeller mass corrected for the effect of the } \\
& \text { entrained water, 0brien [6]; } \\
& \mathrm{b}= \text { length of the cantilever span, } \\
& \theta_{\mathrm{BB}}= \text { the part of the slope of the elastic line at point } A \\
& \text { contributed by the rest of the shaft. }
\end{aligned}
$$

Inertia moment of the propeller mass caused by the acceleration due : : to the slope $\theta_{A}$ alone,

$$
=\left(I_{d} \cdot \ddot{\theta}_{A}+m_{p} \cdot \ddot{y}_{A} \cdot b\right)
$$

From Eq. (12), the deflection at point $\mathrm{A}$ is given by :

$$
y_{A}=\frac{M}{E I} \cdot \frac{b^{2}}{2}
$$

For small deflections we can assume that;

or

$$
\theta_{\mathrm{b}}^{\prime}=\frac{\mathrm{A}_{\mathrm{A}}}{\mathrm{b}}=\frac{\mathrm{M}}{\mathrm{EI}} \cdot \frac{\mathrm{b}}{2}=\frac{\theta_{\mathrm{A}}}{2}
$$

$$
\vdots \quad \mathrm{y}_{\mathrm{A}}=\frac{\mathrm{b}}{2} \cdot \theta_{\mathrm{A}}
$$

where:

$\vdots \quad \theta_{b}^{\prime}=$ the $_{\text {of }}$ part $\underset{A B}{\text { of }}$ slope at point A contributed by the length

Thus:

$$
\begin{aligned}
I_{d} \cdot \ddot{\theta}_{A}+m_{p} \cdot \ddot{y}_{A} \cdot b & =I_{d} \cdot \ddot{\theta}_{A}+m_{p} \cdot \ddot{\theta}_{A} \cdot \frac{b^{2}}{2} \\
& =\left(I_{d}+m_{p} \cdot \frac{b^{2}}{2}\right) \ddot{\theta}_{A}
\end{aligned}
$$

: Elastic moment transmitted by the shaft at $B$

$$
\vdots \quad M=\theta_{B B} \cdot \frac{E \cdot I}{\left(\frac{1}{2}-\frac{g}{3}\right)}
$$

From equations (10) \& (13) the ratios of slopes are:

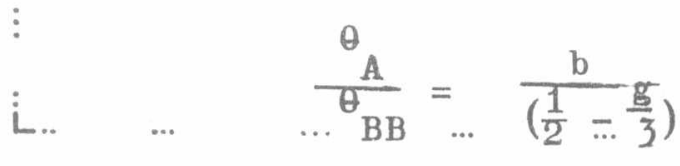


$r$

The equation of motion is thep rewritten as:

$M_{\dot{e}} \sin w t=\left(I_{d}+m_{p} \frac{b^{2}}{2}\right) \cdot \ddot{\theta}_{A}+\left(I_{d}+m_{p} b^{2}\right) \cdot \ddot{\theta}_{B B}+$

$\vdots$

$\quad \theta_{\mathrm{BB}} \cdot\left(\frac{\mathrm{E} \cdot \mathrm{I}}{\frac{\mathrm{l}}{2}-\frac{\mathrm{g}}{3}}\right)$

Substituting for $\theta_{\mathrm{BB}}$, we get :

$\stackrel{\mathrm{M}}{e}_{\mathrm{e}} \operatorname{Sin} w t=\frac{\ddot{\theta}_{\mathrm{BB}} \cdot \mathrm{b}}{\frac{1}{2}-\frac{\mathrm{g}}{3}}\left(I_{d}+\mathrm{m}_{\mathrm{p}} \frac{\mathrm{b}^{2}}{2}\right)+\left(I_{d}+\mathrm{m}_{\mathrm{p}} \mathrm{b}^{2}\right) \cdot \ddot{\theta}_{\mathrm{BB}}+$

$\theta_{\mathrm{BB}}\left(\frac{\mathrm{E} \cdot \mathrm{I}}{\frac{\mathrm{L}}{2}-\frac{\mathrm{g}}{3}}\right)$

$\vdots$

To solve for $\theta_{\mathrm{BB}}$, we assume that;

$$
\begin{aligned}
& \ddot{\theta}_{\mathrm{BB}}=\mathrm{A} \cdot \sin \mathrm{w} t+\mathrm{B} \cdot \cos \mathrm{wt} \\
& \ddot{\theta}_{\mathrm{BB}}=\mathrm{A} \cdot \mathrm{w} \cdot \cos \mathrm{wt}-\mathrm{B} \cdot \mathrm{w} \cdot \sin w t \\
& \ddot{\theta}_{\mathrm{BB}}=-\mathrm{A} \cdot \mathrm{w}^{2} \cdot \sin w t-\mathrm{B} \cdot \mathrm{w}^{2} \cdot \cos w t
\end{aligned}
$$

Substitute equations (22) into equation (21), and equate alike terms, we get:

$M_{e}=-A \cdot w^{2}\left[\left(\frac{b}{\frac{1}{2}-\frac{g}{3}}\right) \cdot\left(I_{d}+m \frac{b^{2}}{2}\right)+\left(I_{d}+m_{p} b^{2}\right)\right]+A \frac{E \cdot I}{\frac{1}{2}-\frac{g}{3}}$.

$0=-B \cdot w^{2} \cdot\left[\left(\frac{\mathrm{b}}{\frac{1}{2}-\frac{\mathrm{g}}{3}} \cdot\left(\mathrm{I}_{\mathrm{d}}+\mathrm{m} p \frac{\mathrm{b}^{2}}{2}\right)+\left(\mathrm{I}_{\mathrm{d}}+\mathrm{m} \mathrm{b}^{2}\right)\right]+\mathrm{B} \quad \frac{\mathrm{E} \cdot \mathrm{I}}{\frac{\mathrm{I}}{2}-\frac{\mathrm{g}}{3}}\right.$

: At resonance, the amplitude of $\theta_{B B}$ is infinite, hence;

$:-w^{2} \cdot\left[\left(\frac{b}{\frac{1}{2}-\frac{g}{3}} \cdot\left(I_{d}+m_{p} \frac{b^{2}}{2}\right)+\left(I_{d}+m_{p} b^{2}\right)\right]+\frac{E}{\frac{I}{2}}-\frac{g}{3}=0\right.$

Finally the natural frequency in cycles per min. is found to be :

$\quad:=\frac{30}{\pi} \sqrt{\frac{E \cdot I}{I_{d} \cdot\left(b+\frac{1}{2}-\frac{g}{3}\right)+m_{p} b^{2} \cdot\left(\frac{b}{2}+\frac{1}{2}-\frac{g}{3}\right)}}$

i. 
$r \cdot \cdots$

The total deflection of the propeller tip is:

$$
\begin{aligned}
& \mathrm{Y}_{\mathrm{pr}}=\mathrm{y}_{\mathrm{A}}+\boldsymbol{\theta}_{\mathrm{BB}} \cdot \mathrm{b} \\
& \therefore \quad=\frac{M b}{E \cdot I} \cdot\left(\frac{b}{2}+\frac{1}{2}-\frac{g}{3}\right)
\end{aligned}
$$

If a further span DE of length-g- is added to the previously studied : system, we get a four span shafting system. Fig. (2).

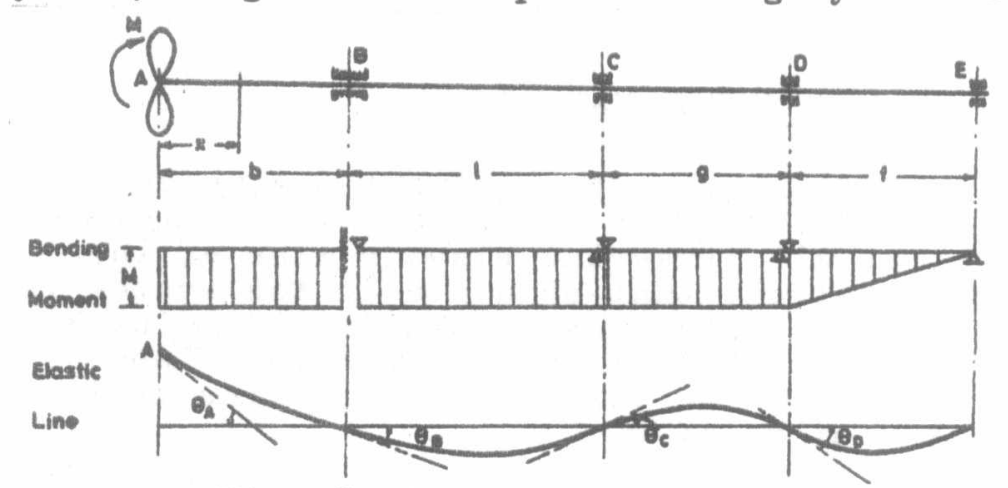

Fig. 2. A System of Four Spans

: starting from the first span to the right-DE- and proceding backwards till we reach the overhanged span -AB-, we would end up with two similar formulae as (24) \& (25).

$\vdots$ Thus the natural frequency in sycles per min and a tip deflection for a four massless span system would be:

$$
\begin{array}{ll}
\quad f=\frac{30}{\pi} \sqrt{\frac{\text { E.I }}{I_{d} \cdot\left(b+\frac{1}{2}-\frac{g}{2}+\frac{f}{3}\right)+m_{p} b^{2} \cdot\left(\frac{b}{2}+\frac{1}{2}-\frac{g}{2}+\frac{f}{3}\right)}} \\
\quad \quad Y_{p r}=\frac{M}{E \cdot I} \cdot b \cdot\left(\frac{b}{2}+\frac{1}{2}-\frac{g}{2}+\frac{f}{3}\right)
\end{array}
$$

GENERAL FORMULA FOR ANY NUMBER OF MASSLESS SPANS

From the similarity of the Eq. (24) \& (26), a general formula could be introduced to calculate the natural frequency of a shafting system of any number of spans.

$$
\vdots \quad \hat{f}=\frac{30}{T} \sqrt{I_{d}\left(L_{0}+\sum_{i=1}^{k}(-1)^{i} \cdot \frac{L_{i}}{2}(-1)^{n} \frac{L^{L}}{3}\right)+m_{p}{ }^{2}\left(\frac{L_{0}}{2}+\sum_{i=1}^{k}(-1)^{i} \frac{L_{i}}{2}+(-1)^{n} \frac{1}{3}\right)} \vdots
$$


The general equation for propeller deflection would:

$\mathrm{Y}_{\mathrm{pr}}=\frac{\mathrm{M}}{\mathrm{E} \cdot \mathrm{I}} \cdot \mathrm{L}_{0} \cdot\left[\frac{\mathrm{L}_{0}}{2}+\sum_{i=1}^{\mathrm{k}}(-1)^{\mathrm{i}} \cdot \frac{\mathrm{L}_{\mathrm{i}}}{2}+(-1)^{\mathrm{n}} \cdot \frac{\mathrm{L}_{1}}{3}\right]$

: where :

$\begin{aligned} & \mathrm{n}=\text { number of total spans studied, } \\ & \mathbf{k}=\mathrm{n}-1 \text {, } \\ & \mathbf{l}=\text { length of overhang, } \\ & \mathrm{L}_{1}=\text { length of the last span, (forward end) }\end{aligned}$

$\mathrm{L}_{i}{ }^{1}={ }_{\text {be always taken equal to zero. }}$ enging in mind that $\mathrm{L}_{1}$ should $\vdots$

$E, I$, and $I_{d}$ are as given before.

$\vdots \quad$ SIIAFT SYSTEMS WITH CONSIDERED MASSES

The following is a method for calculating the natural frequency taking into consideration the effect of the shaft mass. This method based on the assumption that at all times the elastic line of the shaft retains the same configuration, that obtained if the system is: :considered stationary with a pure static moment applied at point A.

This means that the ratios between deflections and slopes at any point of the shaft remain constant. The form of elastic line will : define by knowning the slope $\theta$ at point $B$. $\vdots$

$\vdots$

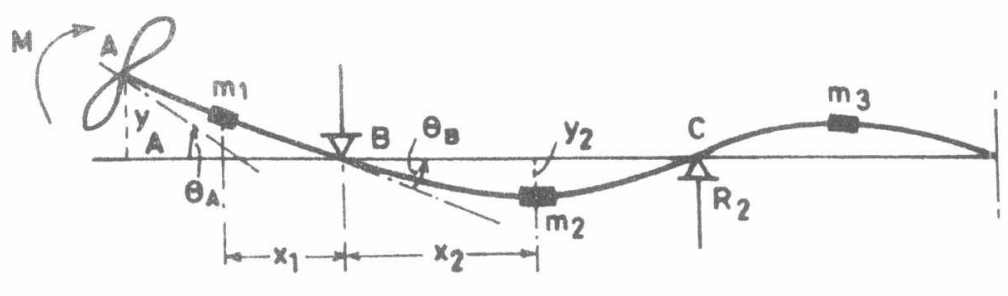

$\vdots$

Fig. (3) Equivalent support beam with effect

of shaft mass taken into consideration

$\vdots$

:From Fig. (3), let $m_{1}$ represent in general the masses between points :

$A$ and $B$ including the distributed mass of the shaft, $x_{1}$ the distance

: of these masses from point $B, m_{2}$ and $x_{2}$ the corresponding

quantities to the right of point $B$.

$\vdots$ 
$r^{\prime}$

Supposing that the application of a static moment $M$ at point $A$ is causing a deformation of the shaft having slope $\theta_{B}$, and the corres-

: ponding slope $\theta_{A}$ and the deflection at the masses $m_{1}$ and $m_{2}$ are in general $y_{1}$ and $y_{2}$. Also let $R_{c}$ represent the reaction force at : the support C. According to the original assumption the ratios

$\frac{\theta_{A}}{\theta_{B}}, \frac{y}{\theta_{B}}$ and $\frac{{ }_{C}}{\theta_{B}}$ remain constant at all times during a vibration cycle.

Taking moments for the entire shaft about point $B$, the differential equation of motion with variable the angle $\theta$ which is the instaneous value of the slope at point $B$.

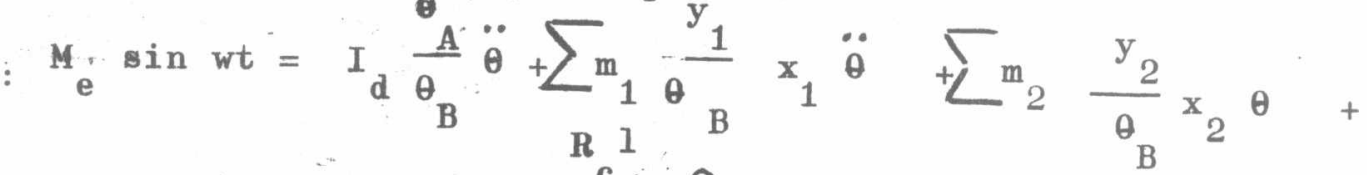

$$
\begin{aligned}
& +\frac{c}{\theta_{B}} \theta
\end{aligned}
$$

where:

$\vdots I_{d}=$ the moment of inertia of the propeller about a diameter. $M^{d} \sin w t=$ the excitation moment

1 = distance of bearing $\mathrm{C}$ from support $\mathrm{B}$.

Eq. (30) can be solved in a manner similar to that of massless

: shaft, and the solution for the natural frequency in cycle per minute will be :

$: \mathbf{f}=\frac{30}{\prod} \sqrt{\frac{R_{c}{ }^{l}}{I_{d}{ }_{\theta}+\sum_{A}{ }_{1} y_{1} x_{1}+\sum m_{2} y_{2} x_{2}}}$

: If more than one span between bearings is to be taken into consider-: ation, the same principle of assuming the configuration of the elastic line remaining proportionalety the same can be accepted, and after the elastic line is drawn for some value of $M$ the quantities

: involved in the equation of the elastic line can be obtained the equation then takes the form:

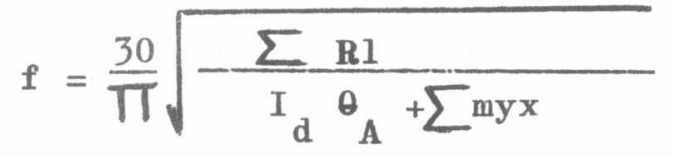

where:

1 = distance of any bearing from support $B$, (Stern-tube bearing)

: $\mathbf{R}=$ the reactions at each support,

$\mathrm{m}=$ mass of shaft element,

$y=$ corresponding deflection of shaft element,

$x=$ distance from each element to support $B$,

i.. 
For the following cases, the numerator and the denominator are analysed separately and then subtituted in Eq. (32) to get the

: fundamental frequency of whirl.

\section{Case of Three Spane}

The propeller mass only causes deflection $\mathrm{y}_{\mathrm{AA}}, \mathrm{Eq} \cdot(25)$ Hence,

$\vdots \quad(\operatorname{myx})_{p}=m_{p} \cdot \frac{M b^{2}}{E \cdot I} \cdot b^{2}\left(\frac{b}{2}+\frac{1}{2}-\frac{g}{3}\right)$

For mass of span $A B$

The equation of deflection:

$\vdots \quad y_{1}=y_{x}+\theta_{B B} \cdot x_{1}$

Using Eq. (12) \& Eq. (10) and substituting for $x=b-x_{1}$, we get; :

$\sum_{m_{1} y_{1} x_{1}}=\mu \frac{M}{1 E \cdot I} \cdot b^{3}\left(\frac{b}{8}+\frac{1}{6}-\frac{g}{9}\right)$

For mass span $\mathrm{BC}$

$y_{2}=\frac{M}{E I}\left(\frac{x_{2}^{2}}{2}+\frac{1 \cdot x_{2}}{2}-\frac{g \cdot x_{2}}{3}\right)$
$\sum_{2} y_{2} x_{2}=\mu_{2} \cdot \frac{M}{E \cdot I} 1^{3}\left(\frac{7}{24} 1-\frac{g}{9}\right)$

For mass of span CD

: Using Eq. (5) \& having $x=x_{3}-1$, we get ;

$\sum m_{3} y_{3} x_{3}=\mu_{3} \frac{M}{E \cdot I}\left(-\frac{7}{360} \cdot g{ }^{4}+\frac{1}{45} \cdot 1 \cdot g^{3}-\frac{1}{20} 1^{2} \cdot g^{2}+\frac{3}{4} 1^{3} g+\frac{1}{6} 1^{4}\right)$

Having defined the terms $I_{d} \& \theta_{A}$ we may conclude the denominator $\vdots$

$$
\begin{aligned}
= & \frac{M}{E I}\left(\left(b+\frac{1}{2}-\frac{g}{3}\right) \cdot I_{d}+m p b^{2}\left(\frac{b}{2}-\frac{1}{2}-\frac{g}{3}\right)+\mu b^{3}\left(\frac{b}{8}+\frac{1}{6}-\frac{g}{9}\right)+1^{3}\left(\frac{71}{24}-\frac{g}{9}\right) \mu_{+}\right. \\
& \left.+\mu\left(\frac{7 g}{360}+\frac{1 g^{3}}{45}-\frac{1^{2} g^{2}}{20}+\frac{31^{3} g}{4}+\frac{1^{4}}{6}\right)\right)
\end{aligned}
$$

where:

$: \mu_{1}, \mu_{2} \mu_{3}=$ mass per unit length of different spans $\mu=$ mass per unit length of all spans where $b, 1, g$, are the equivalent lengths.

: The structure is then salved to calculate the reactions at the different supports.

Hence:

$\vdots \quad \sum \mathbf{R} \cdot L=\mathbf{R}_{\mathrm{C}} \cdot 1+\mathbf{R}_{\mathbf{D}^{\bullet}} \cdot(1+\mathrm{g})$ 
Substituting back in Eq. (38) we can calculate the first frequency of vibration.

Case of Four Spans

The same procedure is applied and the different corresponding

: elements of Eq. ( 32 ) would be :

$$
\begin{aligned}
& (m y x)_{p}=m_{p} \cdot \frac{M b}{E I}\left(\frac{b}{2}+\frac{1}{2}-\frac{g}{2}+\frac{f}{3}\right) \\
& \sum m_{1} y_{1} x_{1}=\mu_{1} \frac{M}{E I} \cdot b^{3}\left(\frac{b}{8}+\frac{1}{6}-\frac{g}{6}+\frac{f}{9}\right) \\
& : \sum m_{2} y_{2} x_{2}=\mu_{2} \frac{M}{E \cdot I} \cdot 1^{3}\left(-\frac{1}{24}-\frac{g}{6}+\frac{f}{9}\right) \\
& \sum_{m_{3} y_{3} x_{3}}=\mu_{3} \cdot \frac{M}{E \cdot I} \cdot g \cdot\left(-\frac{g^{3}}{24}-\frac{25}{12} l^{3}-21 g^{2}+\frac{f l g}{6}+\frac{f g}{9}\right) \\
& : \sum_{4}^{\mathrm{y}_{4} \mathrm{x}_{4}}=\mu_{4} \frac{\mathrm{M}}{\mathrm{E} \cdot \mathrm{I}}\left(\overline{\mathrm{GP}}^{2}-\overline{\mathrm{Gl}}^{2}\right)\left\{-\frac{1}{6 \mathrm{f}}\left[\frac{\overline{\mathrm{Gf}}^{3}-\overline{\mathrm{Gl}}^{3}-\overline{\mathrm{Gl}}^{3}}{5}-\frac{3}{4} \overline{\mathrm{Gl}}\left(\overline{\mathrm{GP}}^{2}-\overline{\mathrm{Gl}}^{2}\right)+\mathrm{f} \cdot \overline{\mathrm{GI}}^{2}\right]+\right. \\
& \left.\vdots \quad \frac{\overline{\mathrm{Gf}}^{2}-\overline{\mathrm{Gl}}^{2}}{8}+\frac{\overline{\mathrm{Gl}}^{2}}{4}-\frac{\mathrm{f} \cdot \overline{\mathrm{Gl}}}{3}-\frac{\mathrm{f}^{2}}{9}-\frac{\mathrm{f} \cdot \overline{\mathrm{G} 1}}{6}\right\} \\
& \text { where; } \overline{\mathrm{Gl}}=\mathrm{g}+1 \\
& \overline{\mathrm{Gf}}=\mathrm{g}+1+\mathrm{f} \\
& \text { The equation for } \theta_{A}=\frac{M}{E \cdot I}\left(b+\frac{1}{2}-\frac{g}{2}+\frac{f}{3}\right)
\end{aligned}
$$

: Then the whole structure is solved to get the values of the nominatoi;$$
\sum \mathbf{R} \cdot L=R_{C} \cdot 1+R_{D}(1+g)+R_{E}(1+g+f)
$$

Finally the frequency equation is found.

\section{REFERENCES}

1. Jasper, N.H., and Rupp, L.A. "An Experimental and Theoretical Investigation of Propeller Shaft Failure". Published SNAME(1952).

2. Solumsmoen, 0.H., "Survey of Vibration Problems". Technical

: Bulletin of Det Norsk Veritas, (1977).

3. Svenson, G., "Shaft Vibration Calculations". Technical Bulletin of Det Norsk Vertas, (1977).

:4. Frivold, H., "Vibration Excitation Forces". Technical Bulletin : of Det Norsk Veritas, (1977).

5. Timoshinko, S., "Strength of Materials" (Part 1), p. 198-209,

: Published By: Affiliated East-West PVT. LTD., (1955).

6. 0'brien, 'T.P., "The Design of Marine Screws Propellers" Published By: The Hutchinson Library of Ships and Shipping, (1962). 
